\title{
$s_{2}-32$ 264307

TDA Progress Report 42-99

November 15, 1989<smiles>O=CC=CC=O</smiles>

\section{Simple Analytic Potentials for Linear Ion Traps}

\author{
G. R. Janik, J. D. Prestage, and L. Maleki \\ Communications Systems Research Section
}

\begin{abstract}
A simple analytical model has been developed for the electric and ponderomotive (trapping) potentials in linear ion traps. This model was used to calculate the required voltage drive to a mercury trap, and the result compares well with experiments. The model gives a detailed picture of the geometric shape of the trapping potential and allows an accurate calculation of the well depth. The simplicity of the model allowed an investigation of related, more exotic trap designs which may have advantages in light-collection efficiency.
\end{abstract}

\section{Introduction}

Radio frequency (RF) quadrupole ion traps have great importance to the development of new atomic frequency standards and high-precision measurements. The threedimensional quadrupole trap has mainly been used. The ideal geometry for the electrodes of this trap is hyperboloids of revolution, which produce a pure quadrupole field. Practical traps are built with a different, more open geometry because of the need to collect light efficiently from the ions. Ion fluorescence is used to determine its quantum state, and the collection efficiency is an important factor in the signal-to-noise ratio. Deviations in the electric field and trapping potential from a pure quadrupole are usually not considered in detail due to the difficulty in calculating them.

In order to increase the number of trapped ions without degrading the frequency stability, the Time and Frequency Systems Research Group recently introduced into frequency-standard research a linear trap based on the quadrupole mass spectrometer [1]. A side benefit of this trap geometry is the ability to calculate the electric and trapping potentials to good accuracy with a simple analytic model. In this article, the model is developed and used to predict an ion resonance frequency, which has been experimentally measured. The article then shows how the model can also be used to investigate similar but more exotic geometries which may be advantageous in some applications.

The linear trap consists of four parallel cylindrical rods arranged with their centers on the corners of a square. An RF voltage is applied to the rods so that nearest neighbors have opposite polarity. This creates an alternating two-dimensional quadrupole electric field between the rods. The field confines ions along the center axis of the trap by the ponderomotive force, just as in a quadrupole mass spectrometer. Two endcap electrodes with a DC bias voltage applied confine the ions axially. This article considers only the center axial region, far enough from the endcaps so that the field is essentially two-dimensional. The DC fields from the endcaps decay exponentially along the axis, 
so that this restriction applies to the vast majority of the trapping volume.

The electric fields due to four cylindrical rods have been studied for years by designers of mass spectrometers and particle accelerators. In order to achieve the closest approximation to a true quadrupole field, it has been found that the ratio of the rod radius to the distance between the rod center and the trap axis reaches its optimum value at 0.5342 . This value was first determined by measurements on a quadrupole accelerator magnet [2] and later reproduced with numerical calculations [3].

A radius-spacing ratio of 0.5342 produces a geometry that is too closed to allow efficient light collection from ions. A pure quadrupole field is not really necessary if ion confinement is the only goal; hence, in this case the ratio can be reduced. The trap discussed here has a ratio of 0.25 , and even smaller ratios might be desirable. The smallness of the ratio leads directly to a simple approximation, namely, the fields produced by infinitely thin rods. A conducting rod with an applied voltage has some induced charge, and as the rod diameter is reduced, all the charge coalesces into a line. This idea can be used to transform a two-dimensional boundary-value problem into a much simpler calculation of the potential produced by fixed sources. All the calculations that follow are based on fields generated by an array of uniform parallel line charges.

\section{Model for a Four-Rod Trap}

Since the model field is two-dimensional, the method of complex variables can be used. This is not necessary, but makes the calculation of the trapping potentials a little easier. Adopting the notation of Landau and Lifshitz [4], a positive line charge at $z=z_{0}$ produces a complex potential $w=-\log \left(z-z_{0}\right)$, whose real part is the ordinary electric potential. Dimensionless quantities are now used to calculate the geometric form of the potentials. Scale factors are introduced later to calculate real trap parameters. The model for the quadrupole trap consists of two negative line charges at $z= \pm i$ and two positive line charges at $z= \pm 1$. This produces the complex potential

$$
w=\ln \left(\frac{z^{2}+1}{z^{2}-1}\right)
$$

The ponderomotive trapping potential is proportional to the square of the electric field $F[5]$. To calculate this, note that $-F_{x}+i F_{y}=d w / d z$, so that

$$
|F|^{2}=\left|\frac{d w}{d z}\right|^{2}=\frac{16|z|^{2}}{\left|z^{4}-1\right|^{2}}
$$

Changing to polar coordinates, it is found that

$$
|F|^{2}=\frac{16 r^{2}}{r^{8}-2 r^{4} \cos 4 \phi+1} \equiv \Gamma G(r, \phi)
$$

where $\Gamma=16$. One can expand $G$ about the origin to obtain

$$
G(r, \phi) \approx r^{2}\left[1+2 r^{4} \cos 4 \phi+r^{8}(1+2 \cos 8 \phi)+\cdots\right]
$$

where the leading term $r^{2}$ is an isotropic harmonic potential, and the higher-order terms have at least fourfold symmetry. It turns out that any two-dimensional field configuration that vanishes at a point produces an isotropic harmonic trapping potential in lowest order about that point. In the trap configurations analyzed later, the function $G$ is always defined to have a leading term $r^{2}$, and $\Gamma$ is used for the numerical factor.

The trapping potential model function $G(x, y)$ is shown in a three-dimensional plot in Fig. 1, and shows a center well rising up to singularities at the rods. Halfway between the rods are saddle points beyond which the potential drops again. The height of the saddle points sets the maximum energy an ion can have and still stay trapped, i.e., the well depth. The well depth can be calculated by setting $\phi=\pi / 4$ in Eq. (1) and finding the maximum value of $G(r, \pi / 4)$. The maximum occurs at $r_{s}=3^{-1 / 4}=0.760$, and has a value of $G_{s}=9 / 16 \sqrt{3}=0.3248$, where the subscript $s$ is the saddle point.

Now that the electric and trapping potentials produced by four line charges have been calculated, a real trap may be modeled. The electric equipotentials of the model are plotted as contours in Fig. 2. Close to the charges, they have a nearly circular shape. The equipotentials of a real trap are exactly circular at the electrode surfaces. The deviation of the model's equipotential from circularity is calculated by finding its horizontal and vertical "diameters" and taking the difference. The electric potential $V$ is the real part of $w$, and is given by

$$
e^{2 V}=\frac{x^{4}+y^{4}+2\left(x^{2}-y^{2}+x^{2} y^{2}\right)+1}{x^{4}+y^{4}+2\left(y^{2}-x^{2}+x^{2} y^{2}\right)+1} \equiv B^{2}
$$

The equipotential surrounding the charge at $z=1$ is to be examined here, so first the intersection of the equipotential with the $x$-axis is found by using

$$
e^{V}=\frac{\left|1+x^{2}\right|}{\left|1-x^{2}\right|}=B
$$


For a fixed value of $V$, Eq. (3) has two positive and two negative solutions. If the smallest positive solution is denoted $x_{0}$, and the other positive solution is denoted $x_{1}$, then Eq. (3) produces the following relationships:

$$
\begin{gathered}
x_{1}=\frac{1}{x_{0}}=\sqrt{\frac{B+1}{B-1}} \\
d \equiv x_{1}-x_{0}=\frac{1-x_{0}^{2}}{x_{0}}=\frac{2}{\sqrt{B^{2}-1}} \\
c \equiv \frac{x_{0}+x_{1}}{2}=\frac{1+x_{0}^{2}}{x_{0}}=\frac{B}{\sqrt{B^{2}-1}}
\end{gathered}
$$

which are valid for $B>1$. Notice that there is a symmetry between $B$ and $1 / B$ corresponding to the symmetry between $V$ and $-V$. The case of $B<1$ applies to the region near the charges at $z= \pm i$.

Point $x_{0}$ corresponds to the inner surface of a rod, $x_{1}$ to the outer surface of a rod, $d$ to the horizontal diameter of the rod, and $c$ to the center of the rod. The "rod" defined here is a hypothetical electrode shaped so that four of them will produce a field distribution equivalent to the four line charges. To calculate the vertical diameter, one can use the intercepts $\pm y_{0}$ on the line $x=c$. Using Eq. (2) results in

$$
y_{0}^{2}=\frac{2 B\left(\sqrt{\left(B^{2}+2\right)}-B\right)-1}{B^{2}-1} \approx \frac{1}{B^{2}}
$$

The approximation is very good for $B \geq 2$, and improves as $B$ increases. The fractional difference of the rod diameters, $\left(d-2 y_{0}\right) / d$, scales as $1 /\left(2 B^{2}\right)$, and is consistent with the idea that the equipotentials become more circular as the line charge is approached. A curious feature of the model is evident in Eq. (4c). The center of the rod $c$ depends on $B$ and does not coincide with the line charge at $x=1$. Seen another way, the line charge sits at the geometric mean of the inner and outer surfaces of the rod $\left(x_{0}\right.$ and $\left.x_{1}\right)$ and only approaches the arithmetic mean as $B$ becomes large.

So far, it has been assumed that the boundary is infinitely far away. This is allowed since the potential $V$ in Eq. (2) tends toward zero for large $r$. The fact that the total charge is zero assures this. Denison [3] has numerically calculated the effect of a circular grounded boundary at $r=1.65$ for an optimized quadrupole with a radius-spacing ratio of 0.5342 . He found that the boundary changed the optimum ratio by about 1 percent. Although a more open trap may be more sensitive to a boundary, practical traps will have their boundaries at greater distances than 1.65 . Therefore, boundaries will be ignored in this treatment.

In order to use the model to calculate the parameters of a trap, the "squashed" rods of the model are associated with the round electrodes of the trap, and the linear dimensions are scaled appropriately. Dimensions of the real trap are given in capital letters; model dimensions are in lower case. The distance of the line charges from the trap axis is $A$, and they will not be exactly at the center of the electrodes. The inner and outer $x$-axis intercepts of the trap rods are called $X_{0}$ and $X_{1}$, respectively. Therefore, the dimensionless coordinates are given by

$$
\begin{aligned}
x_{0} & =\frac{X_{0}}{A} \\
x_{1} & =\frac{X_{1}}{A} \\
A^{2} & =X_{0} X_{1}
\end{aligned}
$$

The mercury ion linear trap has dimensions $X_{0}=7.62 \mathrm{~mm}$ and $X_{1}=12.7 \mathrm{~mm}$, implying $A=9.84 \mathrm{~mm}$ (compared to $10.2 \mathrm{~mm}$ for the true center), $x_{0}=0.774, x_{1}=1.29$, and $B=3.99$. The horizontal and vertical diameters of the equivalent model trap rod differ by 3 percent, providing a good approximation to a circle.

In order to test the predictive power of the model, the applied voltage needed for the mercury ion trap is calculcated to produce a natural ion resonance frequency $\omega$ of $2 \pi \times 48.5 \mathrm{kHz}$. For small displacements, the leading $r^{2}$ term in the trapping potential leads to harmonic motion. The ions are detected by amplifying the current they induce on the trap rods at the natural frequency, and the amplifiers are tuned to $48.5 \mathrm{kHz}$. The driving voltage is applied at a frequency $\Omega$ of $2 \pi \times 500 \mathrm{kHz}$ in a balanced mode, so that the peak voltage on two opposing rods is $V_{0}$ and the voltage on the other two rods is $-V_{0}$ with respect to the vacuum system. The balanced drive keeps the trap axis at zero potential, which allows the application of only DC bias to the endcaps. The amplitude of the applied RF voltage is ramped until a resonance signal appears, indicating that the natural frequency of the trap matches the frequency to which the amplifiers are tuned.

Calculating the required applied voltage uses the fact that on the inner surface of the rod $x_{0}$, the electric potential $V$ equals the applied voltage $V_{0}$, so that

$$
\frac{V}{V_{0}}=\frac{\ln \left|\left(1+x^{2}\right) /\left(1-x^{2}\right)\right|}{\ln \left|\left(1+x_{0}^{2}\right) /\left(1-x_{0}^{2}\right)\right|}=\frac{\ln \left|\left(1+x^{2}\right) /\left(1-x^{2}\right)\right|}{\ln B}
$$


and the electric field is

$$
|E|^{2}=\frac{\Gamma V_{0}^{2} G(x, y)}{(A \ln B)^{2}}
$$

The ponderomotive trapping potential $\Psi$ is given by

$$
\Psi=\frac{e^{2} E^{2}}{4 m \Omega^{2}}=\frac{\Gamma e^{2} V_{0}^{2} G(x, y)}{4 m \Omega^{2}(A \ln B)^{2}}
$$

where $m$ is the mass of the ion [5]. Using the harmonic approximation for $G$ leads to an expression for the natural resonance frequency

$$
\omega=\frac{\sqrt{\Gamma} e V_{0}}{\sqrt{2} m \Omega^{2} A^{2} \ln B}
$$

For mercury isotope 199 and the trap parameters given above, Eq. (7) predicts a peak drive volt age of $93.7 \mathrm{~V}$. The experimental value is $100 \pm 5 \mathrm{~V}$, giving agreement almost within experimental error. The well depth can also be calculated using Eqs. (6) and (7) as

$$
\Psi_{s}=\frac{G_{s} m \omega^{2} A^{2}}{2}
$$

yielding a value of $3.00 \mathrm{eV}$ for the parameters here.

\section{Model for a Two-Rod Trap}

The simplicity of this model makes it useful for analyzing other two-dimensional trap geometries. Any system that produces a point with a vanishing electric field can potentially trap ions. The simplest system consists of two rods driven by the same voltage with respect to a distant boundary. This configuration has a zero field point midway between the two rods. A trap of this type was demonstrated with oil droplets by Straubel [6] very early on, and may be of use in frequency standards due to its wider viewing angle. The model for this trap is two equal negative line charges at $z= \pm i$. The complex potential is

$$
w=\ln \left(z^{2}+1\right)
$$

and the geometric form of the ponderomotive potential is

$$
G(r, \phi)=\frac{r^{2}}{r^{4}+2 r^{2} \cos 2 \phi+1}
$$

with $\Gamma=4$. Contours of the electric equipotentials and a plot of the ponderomotive potential are shown in Figs. 3 and 4 . The two saddle points are on the $x$-axis at $r_{s}=1$, and have the value $G_{s}=1 / 4$. The electric potential can be written as

$$
e^{2 V}=x^{4}+y^{4}+2\left(x^{2}-y^{2}+x^{2} y^{2}\right)+1 \equiv B^{2}
$$

One can calculate the intercepts of an equipotential on the $y$-axis around the charge at $z=i$ and obtain relations analogous to those of the four-rod trap:

$$
\begin{aligned}
& y_{0}=\sqrt{1-B} \\
& y_{1}=\sqrt{1+B}
\end{aligned}
$$

for $B<1$. Combining Eqs. (10a) and (10b) to calculate the scaling law for a real trap results in $2 A^{2}=Y_{0}^{2}+Y_{1}^{2}$, where $Y_{0}$ and $Y_{1}$ are the inner and outer surfaces of the rod. For a trap with rods the same size and spacing as two opposing rods of the mercury ion trap, $A=10.5 \mathrm{~mm}$. This time the line charge is more distant than the rod center $(10.2 \mathrm{~mm})$. A calculation of the diameter difference gives a deviation from roundness of 3 percent.

In order to calculate the applied voltage, the surrounding boundary must be taken into account, because the bars must be driven with respect to something. According to Eq. (9) and Fig. 3, the equipotentials for large $r$ become roughly circular with a value of $2 \ln r$, and it is assumed that the boundary follows one of these contours. The potential on the trap axis is zero and the potential on the rod is $\ln B$. The applied voltage will be proportional to $2 \ln r-\ln B$, where $r=R / A$ and $R$ is the radius of the surrounding boundary. The trapping potential can then be written as

$$
\Psi=\frac{\Gamma e^{2} V_{0}^{2} G}{4 m \Omega^{2} A^{2}(2 \ln r-\ln B)^{2}}
$$

The dimensions of the vacuum system correspond to a value of 2.5 for $r$. In order to duplicate the natural resonance of $48.5 \mathrm{kHz}$ with the two-rod trap, a peak drive voltage of $364 \mathrm{~V}$ is necessary, an increase by a factor of 3.9 over the four-rod trap. The well depth is still governed by Eq. (8), and depends on the boundary only through the voltage necessary to maintain $\omega$. The well depth then is $2.63 \mathrm{eV}$. The price paid for an increased viewing angle is a larger drive voltage and a slightly smaller well depth.

An added complication with this trap is that the electric potential at the trap axis is now oscillating with respect to the vacuum system at the drive frequency. This makes an added AC bias to the endcaps necessary to keep them 
at a constant potential above the trap axis. Straubel did not use endcaps, but relied upon fringing fields from the ends of his rods for axial confinement.

\section{Models for a Three-Rod Trap}

Although the viewing angle is larger in the two-rod trap, the trapping point is still between the two rods. It may be desirable for some applications to trap well outside the electrode structure. This becomes possible with a three-rod trap. A simple example of a three-rod trap model consists of a positive line charge at the origin flanked by two equal negative line charges on the real axis at $z= \pm 1$. The resulting complex and ponderomotive potentials are:

$$
w=\ln \left(\frac{z^{2}-1}{z}\right)
$$

and

$$
|F|^{2}=\frac{r^{4}+2 r^{2} \cos 2 \phi+1}{r^{2}\left(r^{4}-2 r^{2} \cos 2 \phi+1\right)}
$$

The electric ficld vanishes at $z= \pm i$, and these are the trapping points. The electric and trapping potentials are shown in Figs. 5 and 6.

If Eq. (11) is expanded about the trapping point $z=i$, then $\Gamma=4$. The expression for $G$ is too complicated to be very useful. The lowest saddle point can be found from Eq. (11) at $r_{s}=\sqrt{2+\sqrt{5}}=2.058$, and $\phi=\pi / 2$ where $G_{s}=0.0217$. This gives a well depth of $200 \mathrm{meV}$ for the parameters used here. The question of the applied voltage is slightly more complicated than in the previous two cases. Since the total charge is not zero, the boundary must again be included in the calculation. There are now three voltages involved: the central rod $V_{1}$, the outer rods $V_{2}$, and the boundary $V_{3}$. The ratios between these voltages can be calculated from the expression for the potential

$$
e^{2 V}=\frac{x^{4}+y^{4}+2\left(y^{2}-x^{2}+x^{2} y^{2}\right)+1}{x^{2}+y^{2}} \equiv B^{2}
$$

and the various intercepts. For the center $\operatorname{rod}\left(B_{1}>1\right)$, the relation between the $B$ value and the dimensionless diameter is

$$
d_{1}=B_{1}\left(\sqrt{1+\frac{4}{B_{1}^{2}}}-1\right) \approx \frac{2}{B_{1}}
$$

and for the outer rods $\left(B_{2}<1\right)$

$$
d_{2}=B_{2}
$$

and

$$
c_{2}=\sqrt{1+\frac{B_{2}^{2}}{4}}
$$

Once again, the intercepts of the outer rod obey the relations of Eq. (5). The boundary is again assumed to conform to a long-range equipotential at dimensionless radius $r$ with value In $r$. All the potentials can be offset by $\ln r$ to keep the boundary at ground and obtain the following form for the applied voltages:

$$
\frac{V_{1}}{V_{2}}=\frac{\ln \left(B_{1} / r\right)}{\ln \left(B_{2} / r\right)}
$$

Using a value of 2.5 for $r$ and the same rod diameters and spacing as before obtains peak applied voltages of $49.4 \mathrm{~V}$ and $-214 \mathrm{~V}$ for $V_{1}$ and $V_{2}$. A suitably tapped transformer could be used to provide these drive voltages. Alternatively, the ratio of the inner and outer rod diameters could be adjusted to force $B_{1} B_{2}=r^{2}$ so that $V_{1}=-V_{2}$. Even with this simplification, an additional $\mathrm{AC}$ voltage is necessary to bias the endcaps as in the two-rod case.

The influence of the boundary can be removed by choosing the total charge to be zero. This can be accomplished by doubling the center charge. Unfortunately, there is no trapping point with this configuration while the charges are in a line. Moving the center charge down along the imaginary axis to $z=-i b$ creates a trapping point at $z=i / b$. The case of $b=1$ has been analyzed, and the results are shown here. The electric and trapping potentials are plotted in Figs. 7 and 8 . The scaling factor $\Gamma$ is $1 / 4$, and the saddle point is on the $y$-axis at $y_{s}=1.839$ with $G_{s}=0.0728$. The electric potential is

$$
e^{2 V}=\frac{x^{4}+y^{4}+2\left(y^{2}-x^{2}+x^{2} y^{2}\right)+1}{\left(x^{2}+y^{2}+2 y+1\right)^{2}}
$$

and the $x$ intercepts of the outer rods still follow the relationships of Eqs. (4) and (5), with $B$ replaced by $1 / B_{1}$ (for $B_{1}<1$ ). The $y$ intercepts of the lower rod obey the relationship below (for $B_{2}>1$ ):

$$
y_{0,1}=\frac{-B_{2} \pm \sqrt{2 B_{2}-1}}{B_{2}-1}
$$


and Eq. (5). Once again, the voltages applied to the rods will not be equal and opposite unless the diameters are adjusted so that $B_{1}=1 / B_{2}$. The endcaps need only DC bias because the potential is zero at the trapping point.

The small value of $\Gamma$, which is $1 / 4$, makes the drive voltage for the outer rods eight times that of the four-rod trap for the same ion resonance frequency. This fact, plus the small well depth, may limit the usefulness of this trap. In both three-rod traps, however, the low saddle point lies only on one side of the trapping region. The potential barrier on the opposite side is much higher. These traps may be useful for trapping macroscopic particles, where gravity plays an important role. The trap rods could be oriented horizontally so that the low saddle point lies above the trapping region.

\section{Conclusions}

This article has presented a simple model for linear ion traps that permits the accurate calculation of trapping parameters and gives a detailed picture of the potentials. The model was used to analyze some new trap geometries, and their advantages and disadvantages were discussed. Each trapping geometry is characterized by the two parameters $\Gamma$ and $G_{s}$, which determine the dependence of its natural resonance frequency and well depth on the applied RF voltage and trap dimensions.

\section{Acknowledgments}

The authors thank C. Greenhall and G. J. Dick for helpful discussions.

\section{References}

[1] J. D. Prestage, G. J. Dick, and L. Maleki, "New Ion Trap for Frequency Standard Applications," J. Appl. Phys., vol. 66, pp. 1013-1017, 1989.

[2] I. E. Dayton, F. C. Shoemaker, and R. F. Mozley, "The Measurement of TwoDimensional Fields, Part II: Study of a Quadrupole Magnet," Rev. Sci. Instr., vol. 25 , pp. $485-489,1954$.

[3] D. R. Denison, "Operating Parameters of a Quadrupole in a Grounded Cylindrical Housing," J. Vac. Sci. Tech., vol. 8, pp. 266-269, 1970.

[4] L. D. Landau and E. M. Lifshitz, Electrodynamics of Continuous Media, Oxford, UK: Pergamon Press, pp. 13-14, 1960.

[5] H. G. Dehmelt, "Radiofrequency Spectroscopy of Stored Ions, I: Storage," Adv. At. Mol. Phys., vol. 3, pp. 53-154, 1967.

[6] H. Straubel, "Kurze Originalmitteilungen," Naturwissenschaften, vol. 42, pp. 506-507, 1955. 


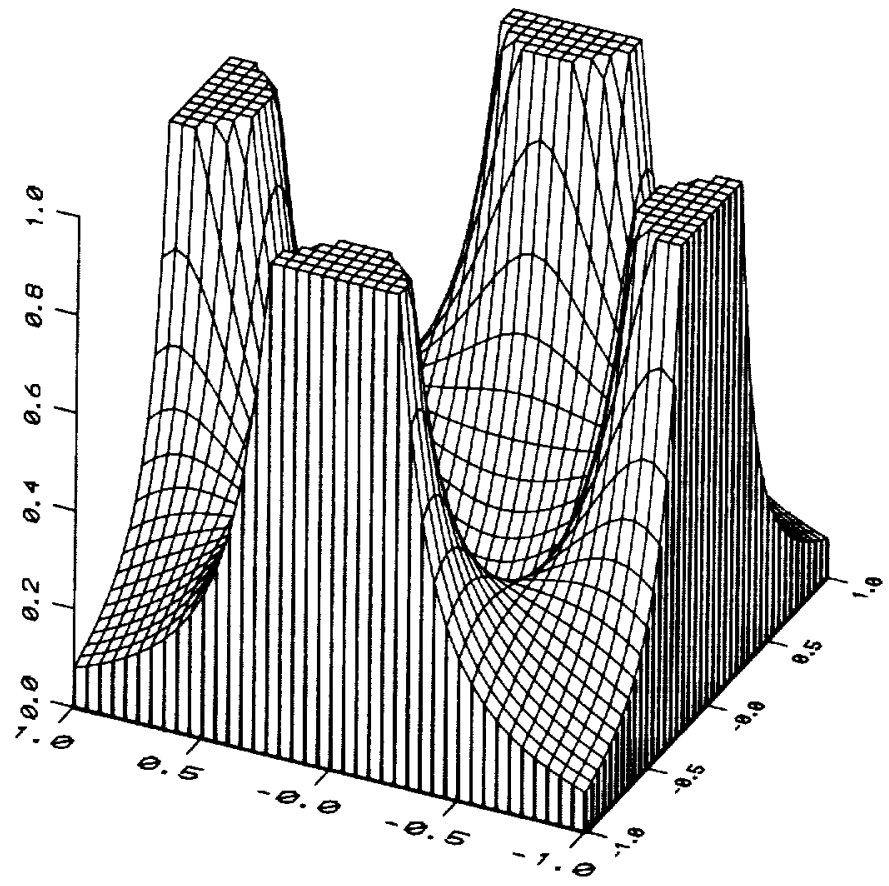

Fig. 1. The ponderomotive potential function $G$ for a four-rod quadrupole trap is plotted in three dimenslons. The singularitles are truncated at the value $\mathbf{1 . 0}$ for clarity.

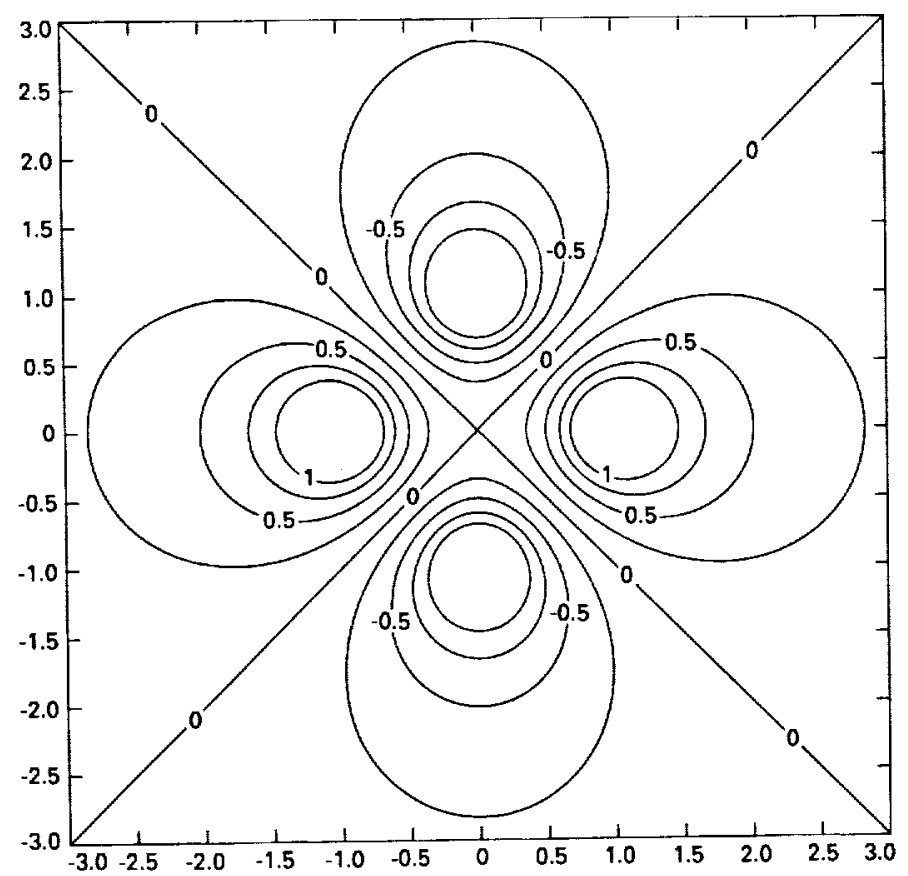

Fig. 2. The electric potential for a four-rod trap is shown in this contour plot. The range of potentlals shown is -1 to 1 at intervals of 0.25 .

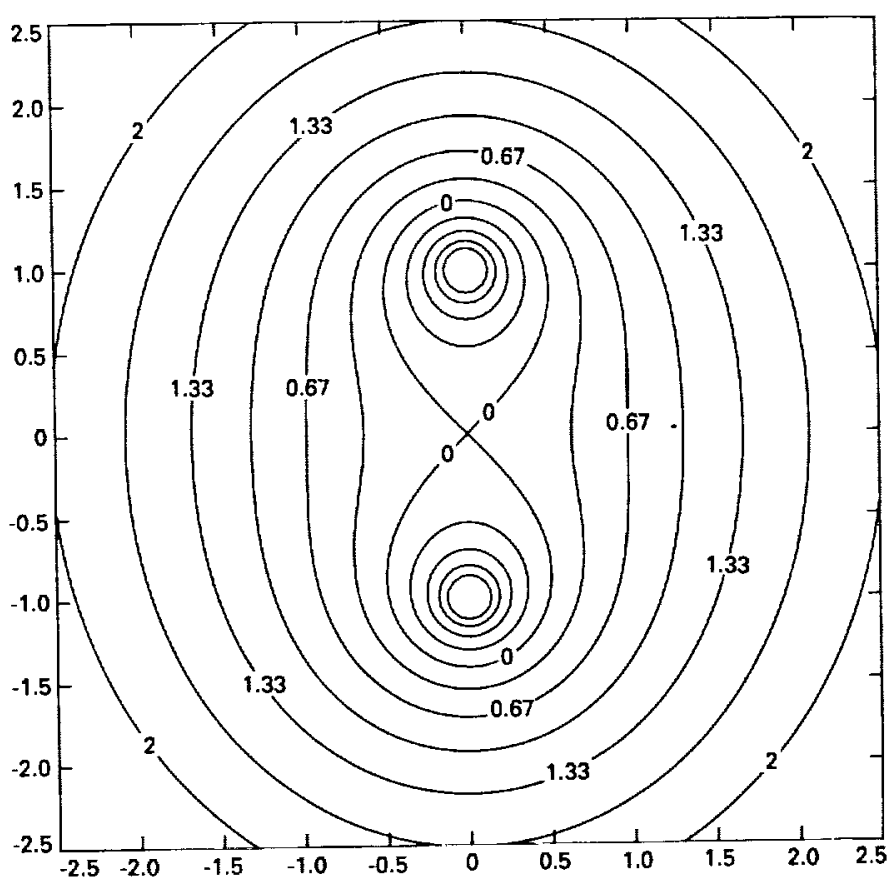

Fig. 3. The electric potential for a two-rod trap is shown in this contour plot. The range of potentlals shown is -1 to 2 at intervals of $1 / 3$.

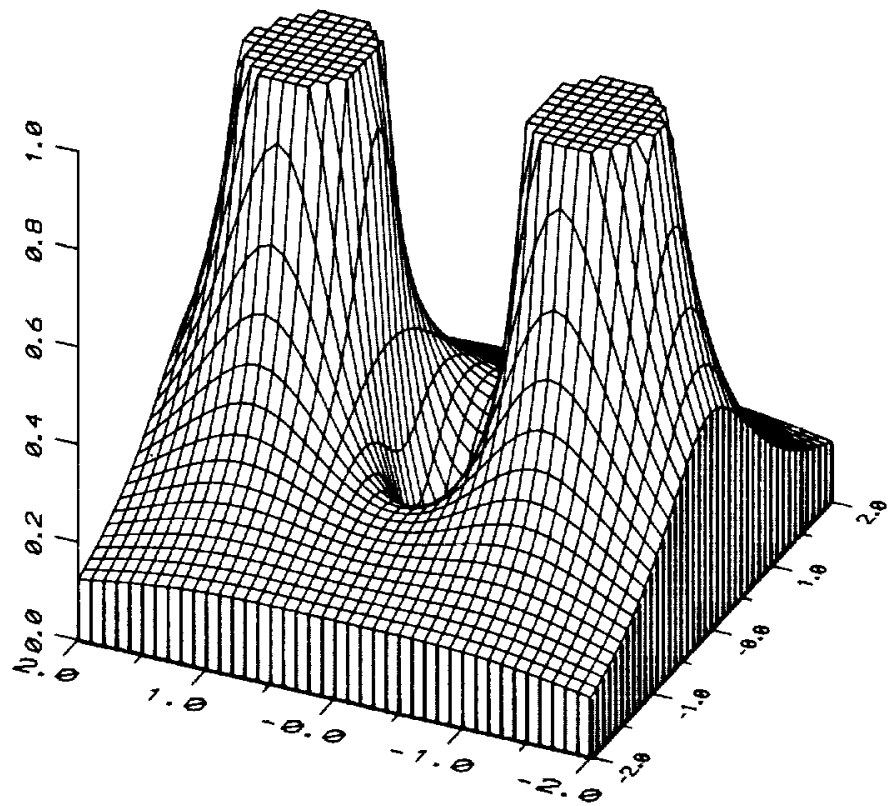

Fig. 4. The ponderomotlve potential function $G$ for a two-rod quadrupole trap is plotted in three dimenslons. The singularities are truncated at the value 1.0 for clarlity. 


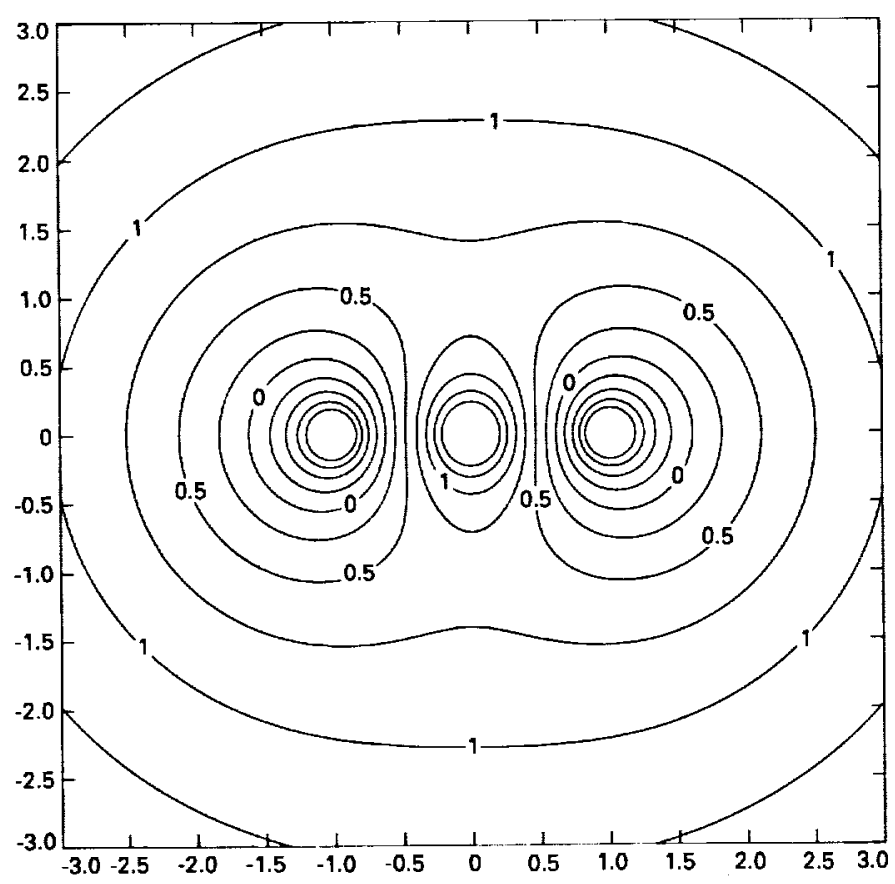

Fig. 5. The electric potentlal for a three-rod trap is shown In this contour plot. The range of potentials shown is -1 to 1.5 at Intervals of $\mathbf{0 . 2 5}$.

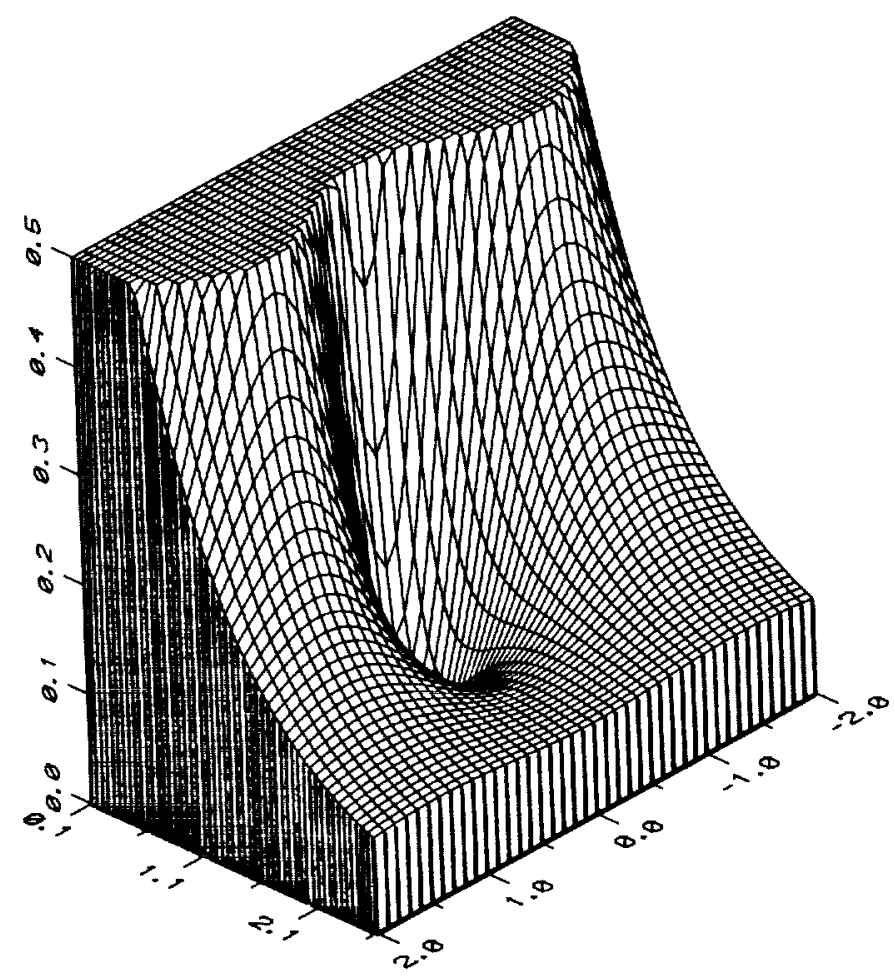

Fig. 6. The ponderomotive potential function $\left|F^{2}\right|$ for a three-rod quadrupole trap is plotted in three dimensions. The singularltles are truncated at the value 0.5 to emphasize the potential well at $z=i$.

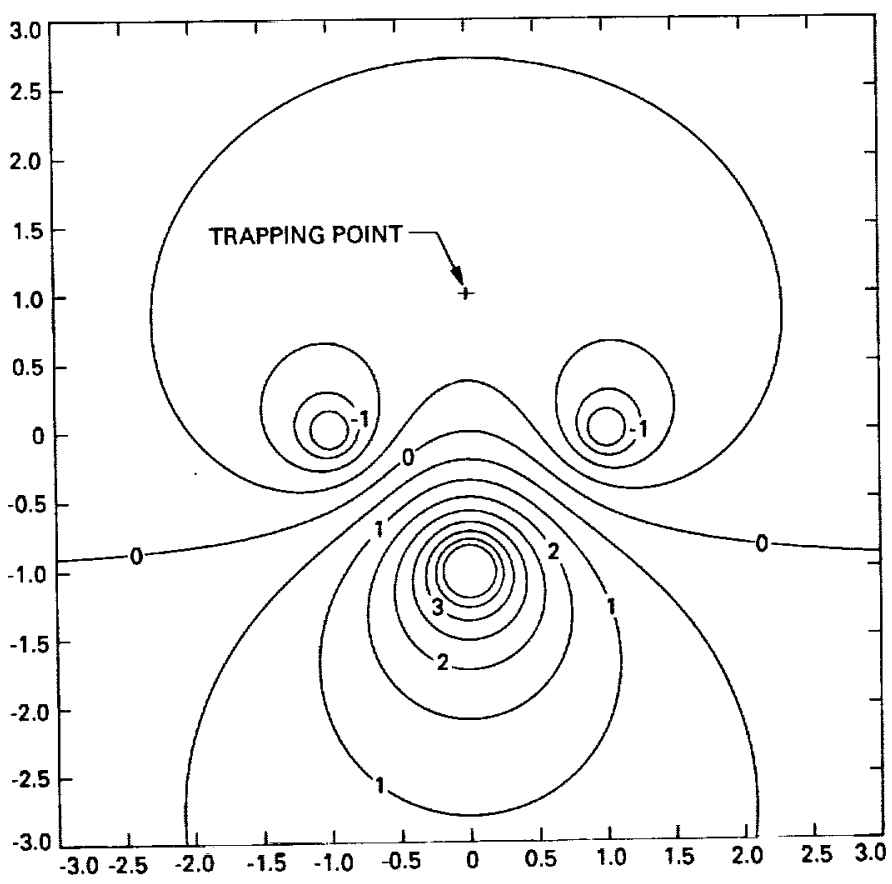

Fig. 7. The electric potential for a three-rod trap with zero net line charge is shown in this contour plot. The range of potentlals shown is -2 to 4 at intervals of 0.5 .

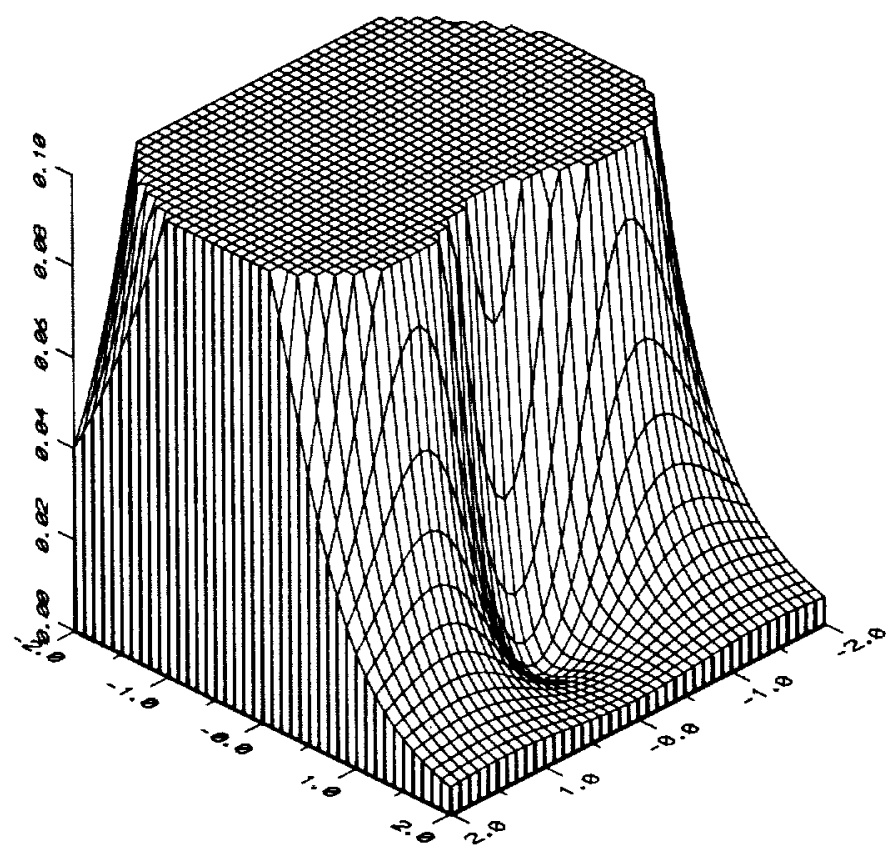

Fig. 8. The ponderomotive potential function $|F 2 / 4|$ for a threerod quadrupole trap with zero net line charge is plotted In three dimenslons. The singularities are truncaled at the value 0.1 to emphasize the potential well at $z=i$. 